\title{
Sur le fonctionnement d'un thermosiphon résultant de la rupture d'équilibre de fluide stratifié
}

\author{
F. Penot et J. L. Peube \\ Laboratoire d'Etudes Aérodynamiques et Thermiques \\ 40, avenue du Recteur-Pineau, 86022 Poitiers, France
}

(Reçu le $1^{\mathrm{er}}$ octobre 1979, révisé le 10 janvier 1980, accepté le 15 janvier 1980)

\begin{abstract}
Résumé. - On discute de la stabilité de l'équilibre hydrostatique d'un fluide chaud contenu dans une conduite courbe dont les extrémités sont situées dans un même plan horizontal. Un critère d'existence d'écoulement stationnaire est obtenu à partir de l'intégration des équations de la couche limite et le fonctionnement du thermosiphon résultant est discuté.
\end{abstract}

\begin{abstract}
We present here a discussion about the stability of hydrostatic equilibrium of a hot fluid contained in an open duct with apertures in the same horizontal plane. An existence criterium of stationary flow is obtained with the help of integration of boundary layer equations and the resulting thermosiphon work is discussed.
\end{abstract}

1. Introduction. - La convection naturelle est un mouvement de fluide dû à l'effet des variations de forces pesantes qui résultent des dilatations provenant des écarts de température au sein du milieu fluide considéré.

Les écoulements correspondants peuvent être rangés en deux grandes catégories :

$1^{\circ}$ pour certains systèmes, le problème tel qu'il est posé avec ses conditions aux limites admet une solution où la température est constante suivant les plans horizontaux.

Cette solution n'est observée que si elle correspond à un équilibre stable. Si cette condition n'est pas réalisée, des mouvements prennent naissance. Les tourbillons de Bénard en sont l'exemple le plus connu.

$2^{\circ}$ pour d'autres, le problème n'a pas de solution à l'équilibre : la température n'est pas constante dans un plan horizontal. C'est le cas, entre autres, d'une plaque plane verticale chauffée placée dans un milieu infini ou des systèmes constitués par un volume de fluide confiné entre deux plaques parallèles verticales et chauffées à des températures différentes [1, 2]

Parmi les écoulements de convection naturelle, les thermosiphons peuvent être définis [3] comme des systèmes de circulation de fluide où l'écoulement est canalisé entre parois de telle sorte qu'on crée une différence de pression motrice due à l'effet cumulatif des poussées d'Archimède sur le fluide confiné. Dans de telles configurations, cet effet global apparaît prédominant, l'écoulement possédant localement des propriétés voisines de celles d'un écoulement de convection forcée. Par exemple, un tube vertical chauffé et ouvert à ses deux extrémités induit un écoulement interne qui peut obéir localement à la loi de Poiseuille. Les écoulements de thermosiphon peuvent appartenir à l'une ou l'autre dés catégories définies précédemment.

L'étude des systèmes de convection naturelle de la première catégorie est bien connue (convection cellulaire de Bénard dans une couche horizontale de fluide chauffé par le bas [4]). La méthode générale consiste à traiter l'instabilité par la théorie des petites perturbations, l'équation intégrale de l'énergie permettant de déterminer l'écoulement existant. Cependant l'étude des thermosiphons de cette catégorie est plus difficile.

La méthode précédente permet, dans quelques cas bien particuliers $[5,6]$, d'aboutir à des solutions, mais en règle générale, tout calcul de stabilité de thermosiphon est rendu impossible car les solutions à l'équilibre font apparaître des discontinuités de température; les thermosiphons possèdent souvent une géométrie complexe. Ils sont de longueur finie et fréquemment ouverts sur un espace infini.

La seule possibilité consiste à trouver des solutions de régime établi à partir d'autres hypothèses (écoulements proches de ceux rencontrés en convection forcée).

Cette étude a pour objet la détermination d'une 
condition d'existence d'écoulement établi dans le cas d'un thermosiphon constitué par une conduite courbe isotherme, telle que ses extrémités débouchent vers le haut et dans un même plan horizontal, dans un fluide plus froid et au repos. La dissymétrie amontaval dans le développement des couches limites dynamiques et thermiques à l'intérieur du conduit constitue l'élément moteur du thermosiphon.

2. Hypothèses et similitude. - Considérons une conduite courbe de section constante chauffée à la température $T_{\mathrm{p}}$. La conduite est ouverte à ses deux extrémités vers un fluide à la température $T_{0}$ (Fig. 1).

En l'absence de toute perturbation, on peut supposer que le fluide contenu dans la conduite se réchauffe jusqu'à la température de paroi et présente au voisinage des ouvertures une stratification de fluide chaud au-dessous de fluide froid. La masse volumique $\rho$ du fluide est alors constante dans un même plan horizontal. La condition d'absence de mouvement :

$$
\operatorname{grad} \rho \wedge \mathbf{g}=0
$$

est donc vérifiée (g étant l'accélération de la pesanteur).

Supposons qu'une perturbation du système entraîne un peu de fluide froid dans l'une des ouvertures. La symétrie verticale est rompue, des forces de poussée d'Archimède prennent naissance d'un côté et ne sont pas compensées de l'autre.

Il en résulte un gradient de pression motrice et par suite un écoulement dans la conduite. Cet écoulement ne peut persister que si les forces de poussée d'Archimède sont suffisamment intenses pour équilibrer l'inertie du fluide et le frottement visqueux.

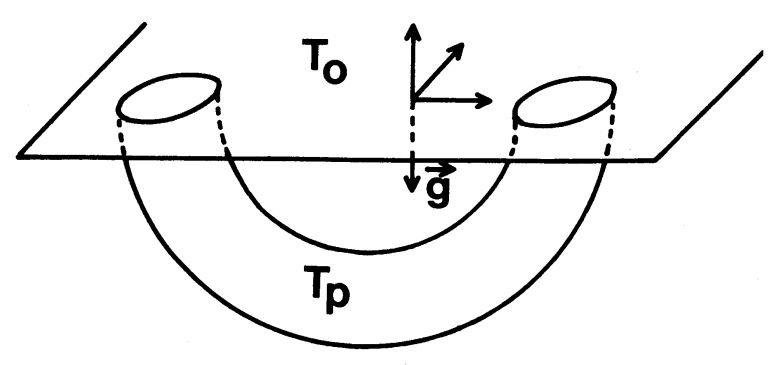

Fig. 1. - Schéma de la conduite.

[Duct scheme.]

Ce mouvement peut alors être décrit par un écoulement de convection forcée (Fig. 2). L'existence d'une longueur d'entrée dans l'une des embouchures de la conduite due au développement des couches limites dynamiques et thermiques est la cause d'une dissymétrie permanente qui entretient le mouvement.

Dans le cas contraire, si les forces de poussée d'Archimède sont insuffisantes, c'est-à-dire si l'écart de température $\left(T_{\mathrm{p}}-T_{0}\right)$ est trop faible, un tel type d'écoulement ne peut s'installer. Le phénomène n'est alors plus régi par les équations de couche limite

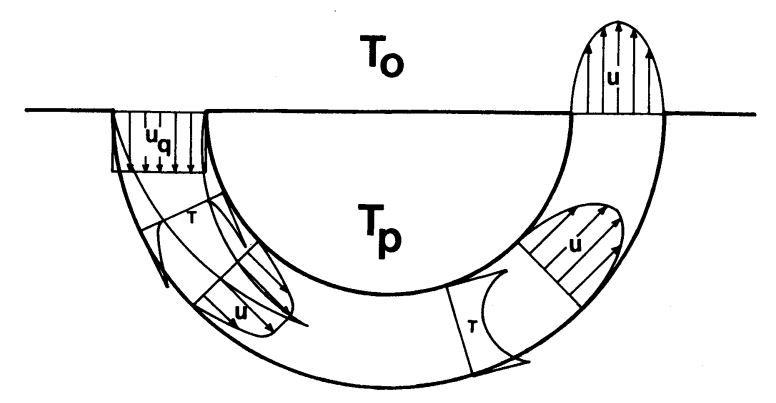

Fig. 2. - Développement des couches limites dans la conduite.

[Duct boundary layers development.]

dans une conduite. L'équilibre est rendu globalement stable.

On suppose que le fluide vérifie les hypothèses de Boussinesq :

- sa masse volumique est constante sauf lorsque ses variations créent des forces volumiques;

- toutes les autres propriétés physiques du fluide sont constantes ;

- la dissipation visqueuse ainsi que les variations de pression sont négligées dans le bilan énergétique effectué à l'aide de l'enthalpie.

Dans ces conditions, pour une géométrie donnée, la similitude montre que le problème dépend de deux paramètres :

- le nombre de Grashof $G r=\frac{\rho^{2} g \beta\left(T_{\mathrm{p}}-T_{0}\right) l^{3}}{\mu^{2}}$

formé avec la longueur $l$ du conduit ;

- le nombre de Prandtl $\operatorname{Pr}=\frac{\mu C_{\mathrm{p}}}{\lambda}$

où $\beta, \mu, C_{\mathrm{p}}, \lambda$ désignent respectivement le coefficient de dilatation, la viscosité, la chaleur massique à pression constante et la conductivité thermique du fluide.

Dans le cadre de l'approximation unidimensionnelle de l'écoulement dans les conduites, la géométrie du système est représentée par la forme de sa ligne moyenne, par une dimension transversale réduite $d / l$ (où $d$ désigne par exemple le diamètre hydraulique de la conduite) ainsi que par des coefficients dynamiques voisins de l'unité qui seront rencontrés dans la suite et qui caractérisent la section droite.

Nous admettrons que le rayon de courbure de la conduite est grand devant son rayon intérieur $(d / l$ est petit devant 1).

De plus, en supposant que la pression est constante dans une section droite et que les variations axiales de vitesse et de température sont petites devant les variations radiales, l'écoulement établi de thermosiphon se place dans le cadre des approximations de la couche limite de convection.

Cette dernière hypothèse exclut les effets centrifuges et la présence de tourbillons de Taylor ou de Goertler [7]. 
3. Equations intégrales. - On définit l'abscisse curviligne $x$ le long de l'axe $O x$ de la conduite, l'origine $x=0$ étant prise dans une des sections d'entrée.

L'équation dynamique projetée sur l'axe $O x$ et l'équation d'énergie s'écrivent respectivement après intégration sur la section droite de la conduite :

$\frac{\mathrm{d}}{\mathrm{d} x} \int_{S} u^{2} \mathrm{~d} s=-\frac{S}{\rho_{0}} \frac{\mathrm{d} p}{\mathrm{~d} x}-\int_{S} g_{x} \beta\left(T-T_{0}\right) \mathrm{d} s-\frac{\chi}{\rho_{0}} \tau_{\mathrm{p}}$

$$
\rho_{0} C_{\mathrm{p}} \frac{\mathrm{d}}{\mathrm{d} x} \int_{S} u T \mathrm{~d} s=\chi q_{T_{\mathbf{P}}}
$$

où $S \quad$ est la section droite de la conduite;

$\chi \quad$ son périmètre ;

$p=p_{0}+\rho_{0} g h$ la pression motrice définie à partir de la masse volumique $\rho_{0}(h$ cote verticale ascendante) ;

$q_{T_{\mathrm{P}}} \quad$ la densité pariétale moyenne de flux thermique dans une section droite;

$\tau_{\mathbf{P}} \quad$ la tension pariétale moyenne de frottement dans une section droite;

$u, T \quad$ la vitesse suivant $O x$ et la température du fluide ;

$g_{x}=+\frac{\partial U}{\partial x}$ la composante suivant l'axe $O x$ de la pesanteur $g$ ( $U$ potentiel du champ de pesanteur).

La vitesse de débit $u_{\mathbf{q}}$, indépendante de l'abscisse $x$, peut être calculée en intégrant les éqs. (1) et (2) entre l'origine $\mathrm{O}$ et l'extrémité de la conduite de longueur $l$ :

$$
\begin{aligned}
& {\left[\int_{S} u^{2} \mathrm{~d} s\right]_{0}^{l}=-\frac{S}{\rho_{0}}[p(l)-p(0)]-} \\
& -\int_{0}^{l}\left[\int_{S} g_{x} \beta\left(T-T_{0}\right) \mathrm{d} s\right] \mathrm{d} x-\frac{\chi}{\rho_{0}} \int_{0}^{l} \tau_{\mathrm{P}} \mathrm{d} x \\
& \rho_{0} C_{\mathrm{p}}\left[\int_{S} u\left(T-T_{0}\right) \mathrm{d} s\right]_{0}^{l}=\chi \int_{0}^{l} q_{T_{\mathrm{P}}} \mathrm{d} x .
\end{aligned}
$$

Posons :

$$
\begin{gathered}
\int_{S} u^{2} \mathrm{~d} s= \\
\alpha u_{\mathrm{q}}^{2} S \int_{S} u\left(T-T_{0}\right) \mathrm{d} s=u_{\mathrm{q}}\left(T_{\mathrm{m}}-T_{0}\right) S \\
\int_{S}\left(T-T_{0}\right) \mathrm{d} s=\gamma\left(T_{\mathrm{m}}-T_{0}\right) S
\end{gathered}
$$

avec $T_{\mathrm{m}}$ température moyenne de mélange

$\alpha, \gamma$ coefficients adimensionnels fonctions de l'abscisse $x$ et de l'ordre de l'unité.

En supposant que la conduite est profilée de telle sorte que la vitesse est uniforme dans la section d'entrée, la pression dans cette section est donnée par le théorème de Bernoulli; soit :

$$
p(0)=-\rho_{0} \frac{u_{\mathbf{q}}^{2}}{2}+p_{0}
$$

$p_{0}$ étant la pression motrice régnant dans l'atmosphère ambiante au-dessus du thermosiphon.

Il vient :

$$
\begin{aligned}
\frac{S}{\rho_{0}} p(l)= & -S \frac{u_{\mathrm{q}}^{2}}{2}+\frac{S}{\rho_{0}} p_{0}-[\alpha(l)-\alpha(0)] u_{\mathrm{q}}^{2} S-S \times \\
& \times \int_{0}^{l} g_{x} \beta \gamma\left(T_{\mathrm{m}}-T_{0}\right) \mathrm{d} x-\frac{\chi}{\rho_{0}} \int_{0}^{l} \tau_{\mathrm{p}} \mathrm{d} x \\
& S \rho_{0} C_{\mathrm{p}} u_{\mathrm{q}}\left[T_{\mathrm{m}}(l)-T_{0}\right]=\chi \int_{0}^{l} q_{T_{\mathrm{p}}} \mathrm{d} x
\end{aligned}
$$

A la sortie de la conduite on applique les conditions de jet en atmosphère libre; en conséquence la pression motrice est égale à $p_{0}$. Par suite on a :

$$
p(l)-p_{0}=p_{\mathrm{T}}(l)-p_{\mathrm{v}}(l)=0
$$

où on a posé :

$P_{\mathrm{v}}$ : perte de pression due à la viscosité et à l'inertie du fluide :

$$
P_{\mathrm{v}}=\frac{\chi}{S} \int_{0}^{l} \tau_{\mathrm{p}} \mathrm{d} x+\rho_{0} u_{\mathrm{q}}^{2}\left[\frac{1}{2}+\alpha(l)-\alpha(0)\right] .
$$

$P_{\mathrm{T}}=$ augmentation de pression due à la température

$$
P_{\mathrm{T}}=-\rho_{0} \int_{0}^{l} g_{x} \beta \gamma\left(T_{\mathrm{m}}-T_{0}\right) \mathrm{d} x .
$$

4. Calcul de $\boldsymbol{P}_{\mathrm{v}}$ et $\boldsymbol{P}_{\mathrm{T}}$ - - La vitesse de débit $u_{\mathrm{q}}$ déterminée par l'éq. (9) constitue l'inconnue du problème. Celui-ci peut être résolu sous une forme adimensionnelle en posant :

$$
R e=\rho_{0} \mathrm{~d} u_{\mathbf{q}} / \mu \text { nombre de Reynolds }
$$

nous pouvons alors effectuer une discussion graphique dans laquelle $R e$ intervient comme paramètre.

Evaluons la perte de pression $p_{\mathrm{v}}$ dans le cas où le régime d'écoulement est laminaire et les profils de vitesse voisins des profils de convection forcée pour que les résultats correspondants soient peu modifiés [8, 9].

En posant :

$$
\begin{aligned}
& P_{\mathrm{T}+}=P_{\mathrm{T}} \rho_{0} d^{2} / \mu^{2} \\
& P_{\mathrm{v}+}=P_{\mathrm{v}} \rho_{0} d^{2} / \mu^{2}
\end{aligned}
$$

l'éq. (10) peut s'écrire alors :

$$
P_{\mathrm{v}+}(l)=\varphi_{1} \operatorname{Re}^{2}+\varphi_{2} \operatorname{Re} \frac{l}{d}\left(1+\varepsilon \operatorname{Re} \frac{d}{l}\right) .
$$


Dans cette expression :

$$
\varphi_{1}=1 / 2+\alpha(l)-\alpha(0)
$$

$\varphi_{1}$ est un coefficient numérique qui dépend de la forme de la section droite de la conduite. $\alpha(l)$ est égal à $4 / 3$ dans le cas d'un écoulement de Poiseuille dans une conduite de section circulaire, $\alpha(l)$ vaut $6 / 5$ pour un écoulement entre deux plans parallèles. Dans le cas où la vitesse est constante dans la section d'entrée, $\alpha(0)=1$.

En conséquence, pour les conduites de sections régulières, $\varphi_{1}$ est voisin de l'unité.

$\varphi_{2}$ est un coefficient de perte de pression régulière dans la conduite. Il est voisin de l'unité. En effet, si $\Lambda$ représente le coefficient de perte de charge dans la conduite, $\varphi_{2}$ est du même ordre de grandeur que $\Lambda \operatorname{Re}(\Lambda=64 / \operatorname{Re}$ dans le cas du tube de section circulaire, $\Lambda=24 / \operatorname{Re}$ dans le cas du canal rectangulaire étroit).

$\varepsilon$ est un coefficient de perte de pression supplémentaire dû aux phénomènes de longueur d'entrée.

La courbe représentative de la fonction $P_{\mathrm{v}}(R e)$ est une fonction croissante qui tourne sa concavité vers le haut (Fig. 3).

Le calcul de l'intégrale $P_{\mathrm{T}}$ nécessite la connaissance de la loi de température $\left(T_{\mathrm{m}}-T_{0}\right)$ en fonction de l'abscisse $x$.

La température sur l'axe de la conduite reste constante et égale à $T_{0}$ sur une longueur $L_{\mathrm{T}}$ (longueur d'entrée thermique) où $\left(T_{\mathrm{m}}-T_{0}\right)$ est une fonction croissante de $x$. Au-delà de cette valeur la température sur l'axe augmente progressivement et la répartition de $\left(T_{\mathrm{m}}-T_{0}\right)$ est toujours une fonction croissante de $x$, jusqu'à une certaine valeur de $x$ égale à $L_{\mathrm{R}}$ pour laquelle elle est pratiquement égale à $\left(T_{p}-T_{0}\right)$. Le fluide est alors complètement réchauffé.

Cependant, l'intégrale fait intervenir le facteur $g_{x}$ qui est positif et décroissant dans la partie descendante de la conduite, négatif dans la partie montante et prend la valeur $-g$ à $x=l$.

$L_{\mathrm{T}}$ et $L_{\mathrm{R}}$ dépendent directement du nombre de Reynolds à un facteur près qui est de l'ordre du

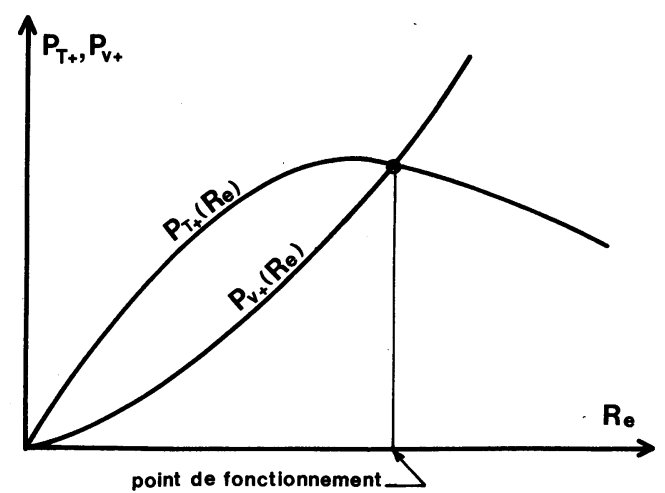

Fig. 3. - Variation de $P_{\mathrm{T}}$ et $P_{\mathrm{v}}$ en fonction du nombre de Reynolds. [ $P_{\mathrm{T}}$ and $P_{\mathrm{v}}$ versus Reynolds number.] nombre de Prandtl. Aux faibles valeurs de $R e, L_{\mathrm{T}}$ et $L_{\mathrm{R}}$ sont petits, $g_{x}$ peut être considéré comme constant, la forme de la courbe représentative de $P_{\mathrm{T}+}(R e)$ est une droite.

Lorsque le nombre de Reynolds augmente $P_{\mathrm{T}+}(R e)$ présente un infléchissement dû à la décroissance de $g_{x}$.

$P_{\mathrm{T}+}$ est donc une fonction croissante puis décroissante du nombre de Reynolds (Fig. 3).

Il vient alors :

$$
P_{\mathrm{T}+}(l)=G r \frac{d^{3}}{l^{3}} \operatorname{Re} \psi_{1} \psi_{2}
$$

$\psi_{1}$ est un coefficient numérique qui tient compte des variations de $g_{x}$ (c'est un terme de la forme $\cos \left(\pi L_{\mathrm{T}} / l\right)$ dans le cas d'une conduite de courbure constante).

$\psi_{2}$ est lié à la loi de variation de la longueur d'entrée en fonction du nombre de Reynolds, $\psi_{2}$ est de l'ordre de l'unité $\left(\psi_{2}\right.$ est voisin de 0,1 en convection forcée avec $\operatorname{Pr}=1)$.

\section{Condition d'existence de mouvement établi. -} Il y a mouvement établi lorsque les courbes $P_{\mathrm{T}+}$ et $P_{\mathrm{v}}+$ se coupent en un point autre que l'origine des axes. La valeur de $R e$ au point d'intersection constitue la solution de (9). On peut remarquer que cette valeur de $R e$ correspond à une solution d'équilibre stable, tandis que la valeur $R e=0$ est une solution d'équilibre instable.

L'existence de ce point d'intersection nous est donnée par une condition sur les pentes à l'origine des deux courbes. A la limite, on obtient le nombre de Grashof critique d'existence du mouvement lorsque :

$$
\left(\frac{\partial P_{\mathrm{T}+}}{\partial R e}\right)_{R e=0}=\left(\frac{\partial P_{\mathrm{v}+}}{\partial R e}\right)_{R e=0} .
$$

Evaluons alors $P_{\mathrm{v}+}$ et $P_{\mathrm{T}+}$ lorsque le nombre de Reynolds est très petit. Dans ce cas, la perte de pression due à la viscosité est équivalente à la perte de pression régulière tout le long de la conduite, on a donc :

$$
P_{\mathrm{v}+}(l)=\varphi_{2} \operatorname{Re}\left(\frac{l}{d}\right)
$$

L'augmentation de pression due à la température est alors équivalente à :

$$
P_{\mathrm{T}+}(l)=\psi_{2} \operatorname{Gr}\left(\frac{d}{l}\right)^{3} R e .
$$

On obtient donc la valeur critique :

$$
\operatorname{Gr}\left(\frac{d}{l}\right)^{4}=\varphi_{2} / \psi_{2}
$$

Si cette condition n'est pas remplie, l'équilibre hydrostatique est rendu stable par l'existence d'une viscosité suffisante, ainsi qu'il est habituel dans ce 
genre de problème (Tourbillons convectifs de Bénard [4, 10]).

On peut estimer la valeur de $G r(d / l)^{4}$ à quelques centaines dans le cas des conduites de section circulaire et quelques dizaines dans le cas des conduites de section rectangulaire et de faible épaisseur.

6. Etude expérimentale. - Une expérience a été mise en œuvre pour l'étude de la convection de l'air entre deux plaques parallèles coudées suivant une demi-circonférence et écartées d'une distance petite devant le rayon de courbure moyen. La conduite ainsi obtenue est fermée latéralement par deux plaques verticales d'un matériau transparent (Fig. 4). Les deux ouvertures sont placées dans un même plan horizontal. Afin de rester dans le cadre de l'évaluation effectuée pour les conduites de section rectangulaire de grande largeur, une conduite de 1,20 m de longueur, de $0,2 \mathrm{~m}$ de largeur et successivement de $0,016 \mathrm{~m}$, $0,020 \mathrm{~m}, 0,028 \mathrm{~m}$ d'épaisseur a été choisie.

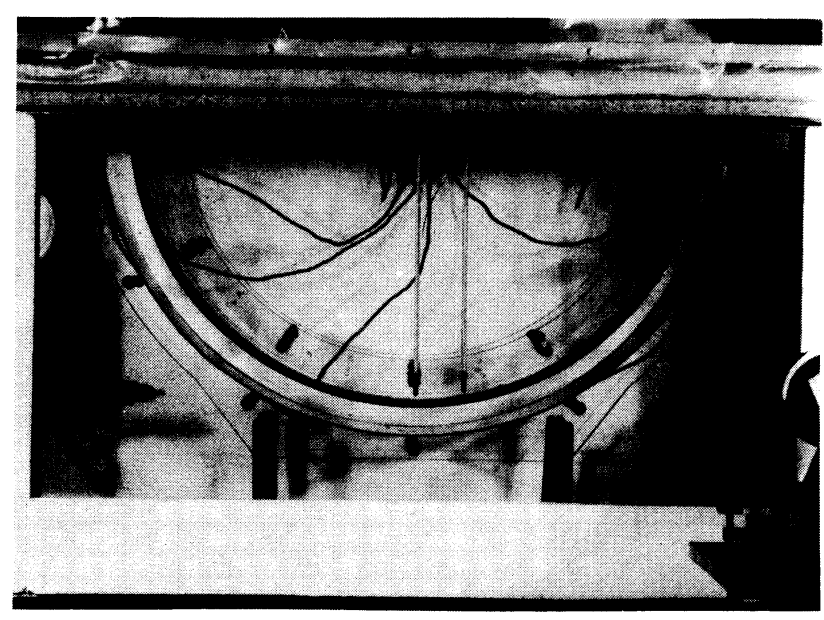

Fig. 4. - Dispositif expérimental.

[Experimental device.]

Les parois de la conduite sont chauffées à une température $T_{\mathrm{p}}$ supérieure à la température $T_{0}$ de l'air ambiant. L'écart $\left(T_{\mathrm{p}}-T_{0}\right)$ maintenu constant prend des valeurs comprises entre 0 et $50^{\circ} \mathrm{C}$.

Des visualisations utilisant du métaldéhyde sublimé en fines particules ont mis en évidence différentes structures d'écoulements :

- Pour des écarts de température $\left(T_{\mathrm{p}}-T_{0}\right)$ inférieurs à $20^{\circ} \mathrm{C}, 8^{\circ} \mathrm{C}, 2,5^{\circ} \mathrm{C}$ (correspondant aux 3 épaisseurs utilisées), on constate l'existence d'une zone de fluide au repos située dans la partie basse de la conduite et qui sépare deux cellules de convection très instables formées par de l'air entrant par une moitié d'une ouverture et remontant vers l'autre moitié de la même ouverture (Fig. 5). La zone de fluide au repos est d'autant plus importante que l'écart $\left(T_{\mathrm{p}}-T_{0}\right)$ est plus faible (relativement à la valeur donnée pour chaque épaisseur de conduite).

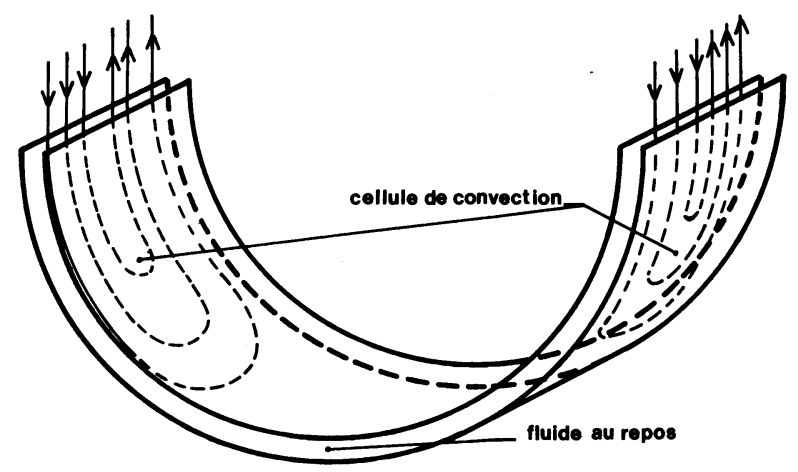

Fig. 5. - Mouvement convectif en deçà de la valeur critique.

[Convective movement under critical value.]

L'écoulement de thermosiphon n'est pas amorcé. L'équilibre hydrostatique est réalisé dans la majeure partie de la conduite. Les poussées d'Archimède sont trop faibles pour entretenir un écoulement global, mais suffisantes pour créer des écoulements cellulaires secondaires dans la zone de stratification des deux ouvertures.

- Pour des écarts de température supérieurs, un écoulement global de thermosiphon, du type envisagé dans les paragraphes précédents, s'installe de lui-même dans la conduite. Il n'a pas de sens préférentiel au départ, mais une fois amorcé il reste toujours dans le même sens. L'expérience répétée pour les différents écartements de plaques conduit à une valeur de $\operatorname{Gr}(d / l)^{4}$ critique de l'ordre de 12,5 .

- Enfin pour des écarts de température plus importants, de l'ordre de $12^{\circ} \mathrm{C}$ pour la conduite de $0,028 \mathrm{~m}$ d'épaisseur et $40^{\circ} \mathrm{C}$ pour celle de $0,020 \mathrm{~m}$ d'épaisseur (il faudrait des écarts de température beaucoup plus grands pour la conduite de $0,016 \mathrm{~m}$ d'épaisseur) il y a formation d'un petit tourbillon qui se développe dans la partie descendante de la conduite et qui reste attaché à la paroi supérieure. Les forces de poussée d'Archimède qui sont localement contraires induisent des phénomènes de convection mixte, caractérisés par un épaississement rapide des couches limites jusqu'à obtenir le décollement pour $G r(d / l)^{4}$ égal à 60 .

7. Conclusion. - Nous avons montré qu'un écoulement établi de thermosiphon n'existe dans la conduite que si le paramètre $\operatorname{Gr}(d / l)^{4}$ dépasse une valeur critique. Une première approximation consistant à considérer l'écoulement proche d'un écoulement de convection forcée a permis, pour des géométries simples, d'en évaluer un ordre de grandeur. Ce paramètre est voisin de 100 pour une conduite de section circulaire, de 10 pour une conduite de section rectangulaire de grand allongement. Ce dernier résultat a été confirmé grâce à une expérience simple de visualisation des structures d'écoulements de l'air dans une conduite courbe. 
En se donnant la forme des profils de vitesse et de température, il est possible d'utiliser le principe de la méthode pour calculer la valeur critique de ce paramètre dans des conduites de formes diverses ainsi que le débit du thermosiphon aux plus grands nombres de Grashof.

Cependant les visualisations ont montré qu'il existe des phénomènes de convection mixte qui deviennent importants aux grands nombres de Grashof. Tenir compte de ces effets est alors à la limite du domaine d'application de la présente méthode qui perdrait de sa simplicité de mise en œuvre.

L'analyse du problème doit dans ce cas être reprise avec des hypothèses améliorées pour la détermination des grandeurs physiques caractérisant le thermosiphon.

\section{Bibliographie}

[1] JANNOT, M., MAZEAS, C., Etude expérimentale de la convection naturelle dans les cellules rectangulaires verticales. Int. J. Heat Mass Trans. 16 (1973).

[2] Aung, W., Fletcher, L. S., Sernas, Y., Developing laminar free convection between vertical plates with asymmetric heating. Int. J. Heat Mass Trans. 15 (1972).

[3] JAPIKSE, D., Advances in thermosiphon technology (Academic press, Advances in heat transfer). Vol. 9, 1973.

[4] ChANDRASEKAR, Hydrodynamic and hydromagnetic stability (Clarendon Press) 1968.

[5] ORHAN KuRAL, The stability of a fluid in a rectangular duct with an adverse temperature gradient. Turbulent buoyant convection, 1976 International seminar, Dubrovnik.

[6] Merker, G. P., Grigull, U., Stability of a convection flow in a shallow cavity with and without rotation. Turbulent buoyant convection, 1976 International seminar, Dubrovnik.

[7] Schlichting, H., Boundary layer theory. 4th edition (Pergamon Press) 1968.

[8] Metaix, B., Eckert, E., Forced, mixed and free convection regime. Journal of heat transfer. Trans. ASME. Ser. E (1964).

[9] Pethukov, B. S., Polyakov, A. F., Flow and heat transfer in horizontal tubes under combined effect of forced and free convection, 4th International heat transfer conference, Versailles, sept. 1970.

[10] Chia ShUN YIH, Fluid mechanic (MacGraw-Hill book company) 1969. 